\title{
Estudio de la especificidad de vías de insulina/IGF-1 en los patrones de arborización y señalización mediante el uso de virus asociados a adenovirus
}

\author{
ALBERTO RIBES-NAVARRO \\ alribes@uji.es \\ ANA SÁNCHEZ PÉREZ \\ sanchean@med.uji.es
}

\section{Resumen}

Objetivo: Las vías de señalización de insulina y del factor de crecimiento insulínico tipo I (IGF-1) están involucradas en el control de la arborización dendrítica, señalización y crecimiento celular. Varios estudios han demostrado que déficits en estas vías están relacionados con trastornos cognitivos, tales como autismo, y con un elevado riesgo de sufrir demencia. Con tal de elucidar el papel exacto que tiene la señalización de insulina cerebral en las funciones neuronales importantes para los procesos cognitivos, pretendemos silenciar la expresión de los substratos del receptor de insulina; IRS-1 e IRS-2, los cuales son dos componentes clave en la vía de señalización de Insulina-IGF-1. Diseño experimental y Métodos: Con tal de estudiar el papel de IRS-1 e IRS-2 en la arborización dendrítica y en la plasticidad neuronal, generaremos varios virus adeno asociados (AAV) que sean específicos de neurona. Además, estas partículas víricas contendrán específicos shRNA para poder silenciar la expresión de IRS-1 y/o IRS-2, junto con un gen chivato para comprobar la eficiencia de la infección del virus. La generación de estas partículas la hemos realizado siguiendo el método de recombinación Gateway, utilizando un promotor específico de neurona y un shRNA contra IRS-1/IRS-2 junto con EGFP como gen chivato. Resultados y conclusiones: Hemos sido capaces de generar una partícula vírica que consta de un promotor genérico potente (CMV) junto con el gen chivato EGFP. Hemos testado la eficiencia y actividad de esta partícula vírica in vivo en varias regiones del cerebro de rata.

Palabras clave: neurobiotecnología, virus adeno asociado, terapia génica, demencia, señalización de insulina en cerebro.

\footnotetext{
Abstract

Objective: The Insulin and insulin-like growth factor 1 (IGF-1) pathway are involved in the normal control of dendritic arborisation, cell signalling and development. Several studies have shown that deficits in these pathways are related to cognitive disorders, such as autism, and an increased risk of dementia. To elucidate the specific role of brain insulin signalling in neuronal functions that are relevant for cognitive processes we want to silence the
} 
gene expression of the Insulin Receptor Substrate 1, and 2, (IRS-1, IRS-2) two key components of the Insulin-IGF-1 pathway. Research Design and Methods: To study the role of IRS-1 and IRS-2 in dendritic arborisation and neural plasticity we want to generate several adeno-associated viruses (AAV) that are neuron-specific. Besides these virus will contain a specific shRNA to silence both IRS-1or IRS-2 and a reporter gene in order to track their infection efficiency. To do so, we have followed the Gateway method for viral particles generation, with a neuron-specific promoter and a shRNA against IRS-1/IRS-2 along with the enhanced green fluorescent protein (EGFP) as a reporter gene. Results and conclusions: We have been able to generate a viral particle that contains a strong generic promoter (CMV) along with a reporter gene (EGFP). We have tested the efficiency and activity of this viral particle in vivo in several regions of the rat brain.

Keywords: neurobiotechnology, adeno associated virus, gene therapy, dementia, brain insulin signalling.

\section{Introducción}

Desde que en la década de los 70 se descubriera el receptor de insulina en cerebro (Havrankova, Schmechel, Roth, \& Brownstein, 1978) una gran cantidad de información ha demostrado que donde antes el cerebro era considerado un órgano independiente de la señalización por insulina y su homólogo, el factor de crecimiento insulínico de tipo I (IGF1), ahora se le reconoce como un centro diana de la acción de estos péptidos.

A nivel de sistema nervioso, las vías de señalización de Insulina e IGF-1 están involucradas en una gran variedad de procesos celulares, incluyendo la arborización dendrítica, crecimiento y reparación celular, regulación de las sinapsis y neuroprotección (Bedse, Di Domenico, Serviddio, \& Cassano, 2015; Craft \& Watson, 2004; Hoyer, 2004; Kleinridders, Ferris, Cai, \& Kahn, 2014; Van Dam \& Aleman, 2004). La insulina juega un papel fundamental en la memoria y el aprendizaje(Bedse et al., 2015) y niveles bajos de IGF-1 circulante y de tanto el receptor de Insulina como de IGF-1 pueden provocar problemas cognitivos y emocionales(Steinman \& Mankuta, 2013; Zhao et al., 2004).

Una de las principales enfermedades causadas por una resistencia a insulina es la diabetes tipo 2; se produce una alteración en el metabolismo de glucosa que resulta en hiperglicemia. Además de la diabetes tipo 2, varios estudios han demostrado que una resistencia a insulina en el cerebro está altamente relacionada con enfermedades neurodegenerativas tales como la enfermedad de Alzheimer (AD) (Talbot et al., 2012).

Los receptores de Insulina (IR) y del IGF-1 (IGF-1R) son glicoproteínas. Cada receptor consiste de dos subunidades a y dos subunidades $\beta$ (Duarte, Moreira, \& Oliveira, 2012; Moloney et al., 2010; Schlessinger, 2000; Wada, Yokoo, Yanagita, \& Kobayashi, 2005). Ambos receptores actúan mediante unión a su ligando, el cuál causa una fosforilación en tirosina del receptor. El receptor fosforilado es activo, une y fosforila el substrato del receptor de insulina (IRS) en residuos de tirosina, lo cual inicia varias cascadas de señalización intracelular que resultan en la activación y modificación de diversos procesos celulares (Duarte et al., 2012).

En cerebro hay dos isoformas de IRS mayormente expresadas: IRS-1 e IRS-2(Sesti et al., 2001). Aunque median las mismas vías y, aparentemente tienen la misma función, los IRS pueden controlar diferentes aspectos de la señalización de Insulina / IGF-1. Los modelos de ratones knockout para estas proteínas muestran que la ausencia de IRS-1 en ratones (IRS-1 - / -) causa 
un crecimiento corporal bajo y una diabetes(Tamemoto et al., 1994). Además, los ratones knockout de IRS-2 tienen una reducción del $50 \%$ en la proliferación neuronal, resultando en una disminución de hasta un $40 \%$ en el tamaño del cerebro. A diferencia de los IRS-1 - / -, los ratones IRS-2 - / - desarrollan diabetes temprana en tres meses de edad, debido a una combinación de resistencia a la insulina periférica y la muerte de las células $\beta$ pancreáticas(Lin et al., 2004; Schubert et al., 2003; Withers et al., 1999). Además, la ausencia de IRS-2 causa infertilidad en ratones hembra (Burks et al., 2000).

Hasta ahora, el uso de animales transgénicos ha sido el método principal para estudiar cómo las diferentes isoformas del sustrato del receptor de insulina pueden afectar las vías de señalización de la insulina y el IGF-1. El gran problema asociado al uso de animales transgénicos es que las alteraciones introducidas en el animal pueden afectar a su desarrollo e incluso pueden Ilegan a ser letales (Burks et al., 2000; Fantin, Wang, Lienhard, \& Keller, 2000; Schubert et al., 2003; Tamemoto et al., 1994). Por último, y muy preocupante es el hecho de que puede haber compensaciones durante el desarrollo que lleguen a enmascarar el efecto de las proteínas en estudio. Una alternativa a la generación de animales transgénicos es el uso de vectores virales que sirvan como vehículo para introducir nuevos genes o alterar la expresión de los genes endógenos en sujetos adultos vivos. Esta técnica abre una nueva gama de posibilidades en el campo de la investigación biomédica, debido a su alta especificidad y versatilidad. Varios estudios han demostrado la efectividad del uso de vectores virales en animales (Alonso et al., 2007; Cascallo et al., 2007; Fueyo et al., 2000; King et al., 2008).

\section{Diseño experimental y Métodos}

\section{Construcción y purificación de partículas víricas derivadas de AAV (rAAV)}

Para la generación de partículas víricas derivadas de AAV se utilizó el método Helper Free de CellBiolabs, INC. Este método permite la generación de AAV recombinantes sin la necesidad de utilizar un Adenovirus como ayudante (helper) para la transfección de nuestra partícula, tal y como se hacía en los métodos convencionales. En este sistema, la mayoría de genes (E2A, E4, y VA) necesarios para la generación de AAV vienen suministrados por un plásmido (que se denomina pHelper, el cual no es un Adenovirus sino un plásmido de DNA). Los genes que codifican para las proteínas de la cápside del AAV (cap, que determinará el serotipo del AAV) y para el ciclo de replicación del AAV (rep) son suministrados en el plásmido pAAV-RC. Finalmente, la propia célula empaquetadora HEK 293 expresa de forma constitutiva el gen E1 de adenovirus. El plásmido de expresión, el cual contiene el promotor genérico CMV y el gen EGFP, se co-transfectó junto con el pHelper y el $p A A V-R C$ en las células empaquetadoras HEK 293 utilizando el método de precipitación con fosfato de calcio, con el cual se obtienen altas eficiencias de transfección. Las células se incubaron duran 72 horas a $37^{\circ} \mathrm{C}$ y 5 $\%$ de $\mathrm{CO}_{2}$. Después de la transfección, las células se recogieron y se lisaron con tal de poder extraer la partícula vírica ya formada. La fracción viral se aisló mediante la precipitación con polietilenglicol (PEG) y cloruro de sodio ( $\mathrm{NaCl}$ ) (Guo et al., 2012; Xiaobing, Xiaoyan, Zhijian, Hong, \& Yunde, 2001). La subsiguiente purificación se llevó a cabo por extracción de cloroformo y diálisis en phosphate buffered saline (PBS) durante toda la noche a $4^{\circ} \mathrm{C}$. Las proteínas víricas se detectaron por tinción de nitrato de plata del gel de poliacrilamida al $12 \%$. La fracción vírica se desnaturaliza previamente durante 10 minutos a $75^{\circ} \mathrm{C}$ en SDS $0,1 \%$; posteriormente la muestra se carga en un gel de acrilamida al $12 \%$ y se detecta su peso molecular mediante tinción por nitrato de plata. 


\section{Estudio in vivo de la infectividad de la partícula vírica}

Para comprobar la actividad de la partícula vírica generada, se utilizaron dos ejemplares hembra de rata Wistar adulta entre $250-350 \mathrm{~g}$. Ambas ratas se mantuvieron en un ciclo de $12 \mathrm{~h}$ de luz y con suministro de comida y agua ad libitum. La partícula vírica se introdujo en el hipocampo y el cuerpo estriado del cerebro de las ratas mediante cirugía esterotáctica, la cual utiliza coordenadas para poder localizar estas regiones en el cerebro. Ambas ratas se anestesiaron con isofluorano, previa cirugía. Se hicieron dos experimentos diferentes: En la primera rata se inyectó una cantidad de $0,5 \mu \mathrm{L}$ de rAAV en hipocampo y $2 \mu \mathrm{L}$ en estriado; esta rata se sacrificó a las 2 semanas de la intervención. A la segunda rata se le inyectó un volumen de $0,3 \mu \mathrm{L}$ de rAAV en hipocampo, $2 \mu \mathrm{L}$ en estriado posterior, y $2,5 \mu \mathrm{L}$ en estriado anterior; sacrificio a las 3 semanas de la intervención.

El sacrificio se realizó mediante inyección letal, posteriormente realizamos la perfusión; el animal se fija en paraformaldehído (PFA) al $4 \%$ y se procede a la extracción del cerebro. El cerebro se crio-protege con una solución de sacarosa al $30 \%$ y se congela. Posteriormente se obtienen cortes de $40 \mu \mathrm{m}$ en el criostato. Estos cortes son posteriormente visualizados al microscopio de fluorescencia para comprobar que se produce señal del EGFP que contenía el virus.

\section{Resultados}

\section{Purificación y titulación del rAVV-CMV-EGFP}

Con tal de comprobar la eficiencia de la co-transfección de nuestros 3 plásmido en las células empaquetadoras HEK 293, éstas se visualizaron al microscopio de fluorescencia (Figura 1). Mediante la visualización al microscopio podemos observar todas aquellas células que hayan incorporado los plásmidos, ya que nuestro plásmido de expresión contiene una secuencia del gen chivato EGFP.

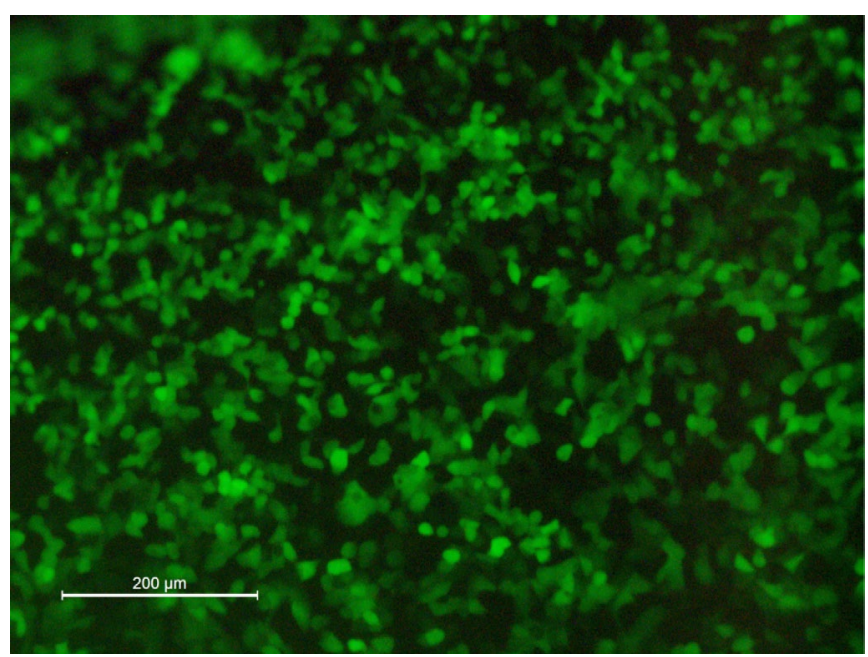

Figura 1. Eficiencia de la co-transfección en HEK 293. Mediante el método del fosfato de calcio se consiguen unas eficiencias de entre el 75 y $80 \%$. Todas aquellas células que presentan fluorescencia son aquellas que han incorporado los plásmido y, por tanto, podrán realizar la encapsulación del virus.

Imagen tomada a 10x aumentos 
Una vez comprobada la eficiencia de la transfección, las células se incubaron durante 72 h y posteriormente, se realizó la extracción y purificación de la partícula vírica. La presencia de rAAV en nuestra muestra purificada se comprobó mediante tinción de nitrato de plata en un gel de acrilamida al $12 \%$ (Figura 2).

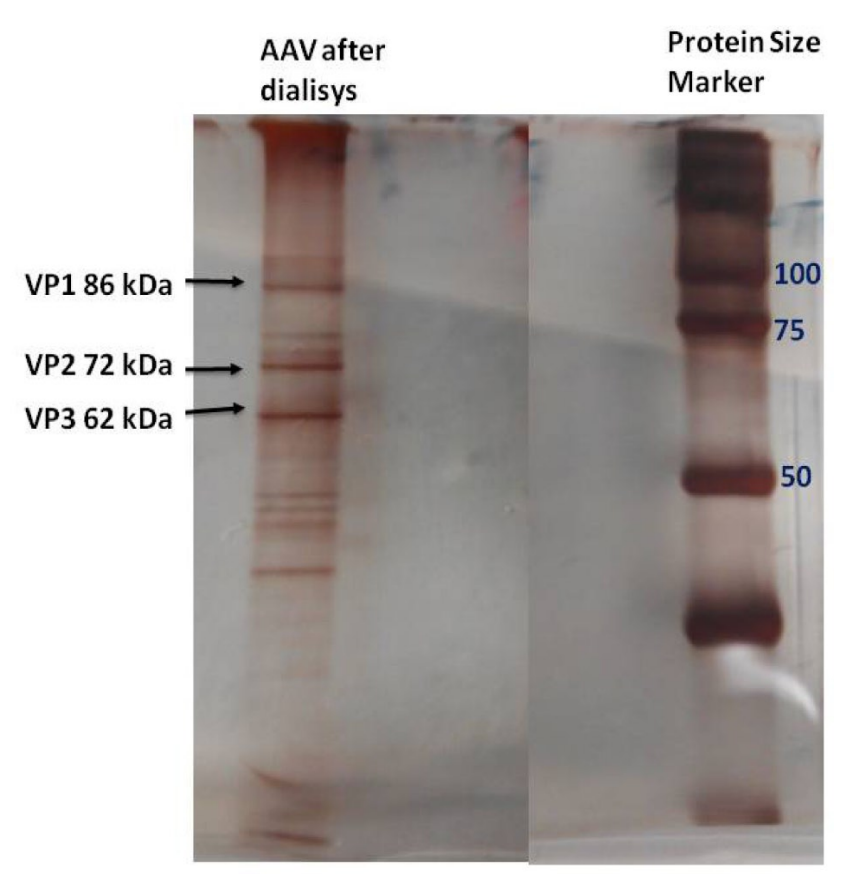

Figura 2. Tinción en nitrato de plata del gel de poliacrilamida al $12 \%$. Las muestras se cargaron en un gel de poliacrilamida para poder detectar el peso molecular de las proteínas características del AAV (VP1, VP2, VP3).

El carril de la izquierda muestra nuestro rAAV purificado, mientras que el carril de la derecha es el marcador de peso molecular Precision Plus Protein ${ }^{\mathrm{TM}}$, Kaleidoscope ${ }^{\mathrm{TM}}$ (Bio-Rad)

\section{Test in vivo rAAV}

Una vez titulada nuestra partícula vírica, se introdujo en cerebro de rata mediante cirugía esterotáctica con tal de testar su eficiencia in vivo. Se realizaron dos experimentos diferentes (véase apartado 2 de Métodos). Hasta el momento sólo se han obtenido datos para el primer experimento, en el cual la rata se sacrificó dos semanas después de la intervención. En este experimento se hicieron dos inyecciones, una de $0,5 \mu \mathrm{L}$ de rAAV en hipocampo y otra de $2 \mu \mathrm{L}$ en estriado (Figura 3). 
A
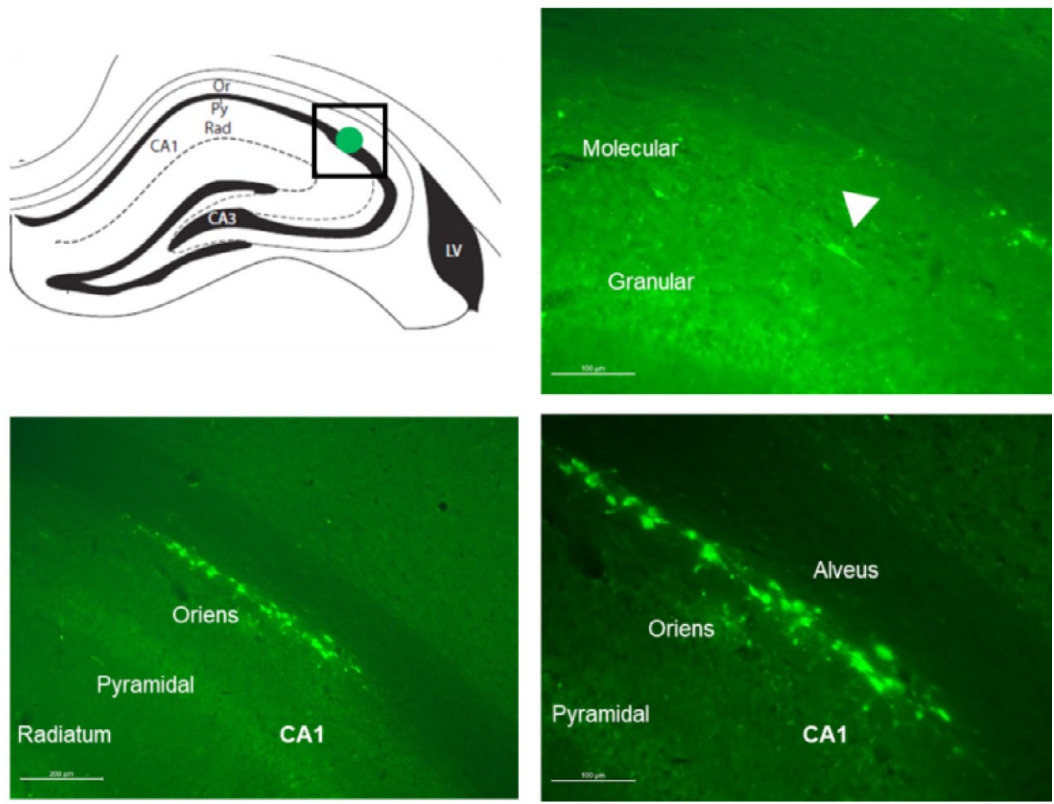

B
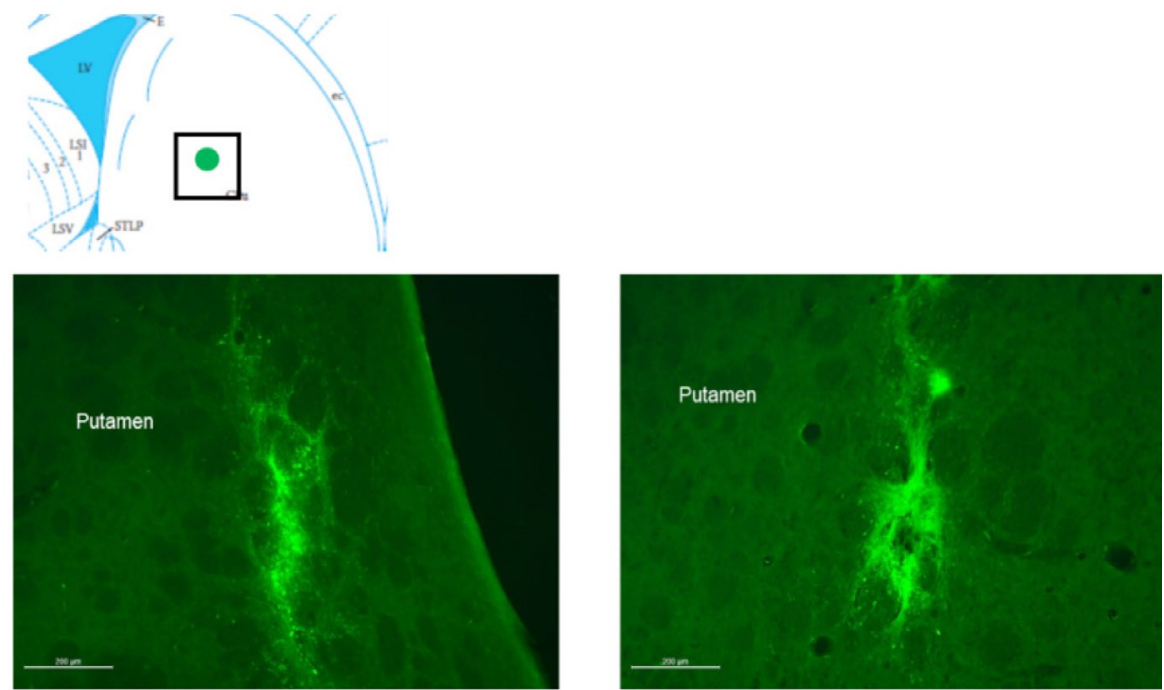

Figura 3. Actividad del rAAV-CMV-EGFP en Hipocampo (A) y Estriado de rata (B). 2 semanas post- inyección los cerebros fueron crio preservados. Posteriormente se hicieron cortes de $40 \mu \mathrm{m}$ para visualización en microscopio de fluorescencia

\section{Discusión y conclusiones}

En este proyecto se ha abordado la posibilidad de generar partículas víricas para el estudio de las vías de señalización de Insulina-IGF-1 como alternativa a la generación de animales transgénicos debido a los problemas que éstos pueden acarrear (Burks et al., 2000; Fantin et al., 2000; Schubert et al., 2003; Tamemoto et al., 1994).

Previa generación de las partículas víricas con las secuencias silenciadoras pertinentes para bloquear la expresión de IRS-1 e IRS-2 se decidió generar una partícula control para poder 
validar el método de generación y purificación de rAAV. Se utilizaron los plásmidos y las células facilitados por CellBiolabs, INC con tal de generar un rAAV control el cual produjera expresión de la proteína fluorescente EGFP bajo el control de un promotor constitutivo (CMV).

Mediante el método de co-transfección con fosfato de calcio y posterior precipitación y purificación del rAAV con PEG/NaCl y subsiguiente diálisis en PBS hemos conseguido generar y obtener la partícula vírica rAAV-CMV-EGFP, la cual es activa in vivo.

Este hallazgo abre una nueva ventana en nuestra investigación, ya que una vez hemos conseguido optimizar el método de generación y purificación de rAAV, podemos empezar a generar diversas partículas víricas bajo el control de promotores específicos de neurona y/o astrocito junto con secuencias silenciadoras tanto para IRS-1 como para IRS-2. De esta forma, podremos estudiar el papel específico que juega cada una de estas moléculas en la señalización de Insulina/IGF-1 en el cerebro.

\section{Referencias bibliográficas}

Alonso, M. M., Cascallo, M., Gomez-Manzano, C., Jiang, H., Bekele, B. N., Perez-Gimenez, A., ... Fueyo, J. (2007). ICOVIR-5 shows E2F1 addiction and potent antiglioma effect in vivo. Cancer Research, 67(17), 8255-8263. https://doi.org/10.1158/0008-5472.CAN-06-4675

Bedse, G., Di Domenico, F., Serviddio, G., \& Cassano, T. (2015). Aberrant insulin signaling in Alzheimer's disease: Current knowledge. Frontiers in Neuroscience, 9(MAY), 1-13. https:// doi.org/10.3389/fnins.2015.00204

Burks, D. J., Font de Mora, J., Schubert, M., Withers, D. J., Myers, M. G., Towery, H. H., ... White, M. F. (2000). IRS-2 pathways integrate female reproduction and energy homeostasis. Nature, 407(6802), 377-82. https://doi.org/10.1038/35030105

Cascallo, M., Alonso, M. M., Rojas, J. J., Perez-Gimenez, A., Fueyo, J., \& Alemany, R. (2007). Systemic toxicity-efficacy profile of ICOVIR-5, a potent and selective oncolytic adenovirus based on the pRB pathway. Molecular Therapy: The Journal of the American Society of Gene Therapy, 15(9), 1607-1615. https://doi.org/10.1038/sj.mt.6300239

Craft, S., \& Watson, G. S. (2004). Insulin and neurodegenerative disease: Shared and specific mechanisms. Lancet Neurology, 3(3), 169-178. https://doi.org/10.1016/S1474-4422(04)00681-7

Duarte, A. I., Moreira, P. I., \& Oliveira, C. R. (2012). Insulin in central nervous system: More than just a peripheral hormone. Journal of Aging Research, 2012. https://doi. org/10.1155/2012/384017

Fantin, V. R., Wang, Q., Lienhard, G. E., \& Keller, S. R. (2000). Mice lacking insulin receptor substrate 4 exhibit mild defects in growth, reproduction, and glucose homeostasis. American Journal of Physiology. Endocrinology and Metabolism, 278(1), E127-33.

Fueyo, J., Gomez-Manzano, C., Alemany, R., Lee, P. S., McDonnell, T. J., Mitlianga, P., ... Kyritsis, a P. (2000). A mutant oncolytic adenovirus targeting the Rb pathway produces antiglioma effect in vivo. Oncogene, 19(June 1999), 2-12. https://doi.org/10.1038/sj. onc.1203251

Guo, P., El-Gohary, Y., Prasadan, K., Shiota, C., Xiao, X., Wiersch, J., ... Gittes, G. K. (2012). Rapid and simplified purification of recombinant adeno-associated virus. Journal of Virological Methods, 183(2), 139-146. https://doi.org/10.1016/j.jviromet.2012.04.004

Havrankova, J., Schmechel, D., Roth, J., \& Brownstein, M. (1978). Identification of insulin in rat brain. Proceedings of the National Academy of Sciences of the United States of America, 75(11), 5737-41.

Hoyer, S. (2004). Glucose metabolism and insulin receptor signal transduction in Alzheimer disease. European Journal of Pharmacology, 490(1-3), 115-125. https://doi.org/10.1016/j. ejphar.2004.02.049 
King, G. D., Muhammad, a K., Curtin, J. F., Barcia, C., Puntel, M., Liu, C., ... Castro, M. G. (2008). Flt3L and TK gene therapy eradicate multifocal glioma in a syngeneic glioblastoma model. Neuro Oncol, 10(1), 19-31. https://doi.org/10.1215/15228517-2007-045

Kleinridders, A., Ferris, H. a., Cai, W., \& Kahn, C. R. (2014). Insulin action in brain regulates systemic metabolism and brain function. Diabetes, 63(7), 2232-2243. https://doi. org/10.2337/db14-0568

Lin, X., Taguchi, A., Park, S., Kushner, J. a, Li, F., Li, Y., \& White, M. F. (2004). Dysregulation of insulin receptor substrate 2 in beta cells and brain causes obesity and diabetes. J Clin Invest, 114(7), 908-916. https://doi.org/10.1172/JCl200422217.908

Moloney, A. M., Griffin, R. J., Timmons, S., O'Connor, R., Ravid, R., \& O'Neill, C. (2010). Defects in IGF-1 receptor, insulin receptor and IRS-1/2 in Alzheimer's disease indicate possible resistance to IGF-1 and insulin signalling. Neurobiology of Aging, 31(2), 224-243. https:// doi.org/10.1016/j.neurobiolaging.2008.04.002

Schlessinger, J. (2000). Cell Signaling by Receptor Tyrosine Kinases A large group of genes in all eukaryotes encode for. October, 103(2), 211-225. https://doi.org/10.1016/j. cell.2010.06.011

Schubert, M., Brazil, D. P., Burks, D. J., Kushner, J. a, Ye, J., Flint, C. L., ... White, M. F. (2003). Insulin receptor substrate-2 deficiency impairs brain growth and promotes tau phosphorylation. The Journal of Neuroscience: The Official Journal of the Society for Neuroscience, 23(18), 7084-7092. https://doi.org/23/18/7084 [pii]

Sesti, G., Federici, M., Hribal, M. L., Lauro, D., Sbraccia, P., \& Lauro, R. (2001). Defects of the insulin receptor substrate (IRS) system in human metabolic disorders. FASEB Journal, 15, 2099-111. https://doi.org/10.1096/fj.01-0009rev

Steinman, G., \& Mankuta, D. (2013). Insulin-like growth factor and the etiology of autism. Medical Hypotheses, 80(4), 475-480. https://doi.org/10.1016/j.mehy.2013.01.010

Talbot, K., Wang, H., Kazi, H., Han, L., Bakshi, K. P., Stucky, A., ... Arnold, S. E. (2012). Demonstrated brain insulin resistance in alzheimer's disease patients is assocaited with IGF-1 resisitance, IRS-1 dysregulation, and cogntive decline. Journal of Clinical Investigation, 122(4). https://doi.org/10.1172/JCI59903DS1

Tamemoto, H., Kadowaki, T., Tobe, K., Yagi, T., Sakura, H., Hayakawa, T., ... Aizawa, S. (1994). Insulin resistance and growth retardation in mice lacking insulin receptor substrate-1. Nature, 372(6502), 182-186.

Van Dam, P. S., \& Aleman, A. (2004). Insulin-like growth factor-I, cognition and brain aging. European Journal of Pharmacology, 490(1-3), 87-95. https://doi.org/10.1016/j. ejphar.2004.02.047

Wada, A., Yokoo, H., Yanagita, T., \& Kobayashi, H. (2005). New twist on neuronal insulin receptor signaling in health, disease, and therapeutics. Journal of Pharmacological Sciences, 99(2), 128-143. https://doi.org/10.1254/jphs.CRJ05006X

Withers, D. J., Burks, D. J., Towery, H. H., Altamuro, S. L., Flint, C. L., \& White, M. F. (1999). Irs-2 coordinates Igf-1 receptor-mediated beta-cell development and peripheral insulin signaIling. Nature Genetics, 23(1), 32-40. https://doi.org/10.1038/12631

Xiaobing, W. U., Xiaoyan, D., Zhijian, W. U., Hong, W., \& Yunde, H. O. U. (2001). NOTES A novel method for purifica- tion of recombinant adeno- associated virus vectors on a large scale. Chinese Science Bulletin, 46(6), 3-6.

Zhao, L., Teter, B., Morihara, T., Lim, G. P., Ambegaokar, S. S., Ubeda, O. J., ... Cole, G. M. (2004). Insulin-Degrading Enzyme as a Downstream Target of Insulin Receptor Signaling Cascade : Implications for Alzheimer's Disease Intervention. Analyst, The, 24(49), 1112011126. https://doi.org/10.1523/JNEUROSCI.2860-04.2004 